\title{
Nematic Quantum Criticality Without Order
}

\author{
H. Yamase, ${ }^{1}$ P. Jakubczyk, ${ }^{2}$ and W. Metzner ${ }^{3}$ \\ ${ }^{1}$ National Institute for Materials Science, Tsukuba 305-0047, Japan \\ ${ }^{2}$ Institute of Theoretical Physics, Faculty of Physics, \\ University of Warsaw, Hoża 69, 00-681 Warsaw, Poland \\ ${ }^{3}$ Max-Planck-Institute for Solid State Research, D-70569 Stuttgart, Germany
}

(Dated: May 25, 2022)

\begin{abstract}
We consider a two-dimensional interacting Fermi system which displays a nematic phase within mean-field theory. The system is analyzed using a non-perturbative renormalization-group scheme. We find that order-parameter fluctuations can suppress the nematic order obtained in mean-field theory even at zero temperature. For a suitable choice of parameters a quantum critical point surrounded by a disordered phase can be realized, giving rise to quantum critical behavior in the absence of an ordered regime in the phase diagram.

PACS numbers: 05.10.Cc, 73.43.Nq, 71.27.+a
\end{abstract}

Classical phase transitions are controlled by an energy-entropy balance, and the critical behavior of continuous transitions is caused by thermal fluctuations. By contrast, quantum phase transitions occur at zero temperature and are driven by quantum effects [1]. In the last decade there has been growing interest in quantum phase transitions in metallic systems, and especially in deviations from conventional Fermi liquid behavior due to quantum critical fluctuations near a quantum critical point [2, 3].

A widely used approach to approximately determine equilibrium phase diagrams of interacting systems is the mean-field theory (MFT). In numerous cases this approach yields a qualitatively correct description of the phase diagram in question. There are however also results revealing the inadequacy of this approximation in certain situations, in particular in low-dimensional systems. The Mermin-Wagner theorem [4] delivers a well-known, transparent and rigorous example of such a result for finite temperatures and spatial dimension $d \leq 2$. A recent example for the case $T=0$ was provided by Greenblatt et al. [7], establishing rigorously the Imry-Ma rounding phenomenon due to disorder for quantum phase transitions. 
Many other (mostly approximate) results point toward the very important role played by quantum fluctuations in $d=2$ and $T \geq 0$ [1 3$]$. Also for a number of systems in $d=3$ and $T \geq 0$ (see e.g. Ref. [5]) fluctuations are known to severely influence the phase diagram, for example by turning a continuous transition into a first-order one, or vice-versa [6].

There is considerable interest in two-dimensional itinerant electron systems with interactions favoring a $d$-wave Pomeranchuk instability [8, 9] leading to a so-called electronic nematic state [10], where the point group symmetry of the underlying square lattice is spontaneously broken. There is growing evidence that a nematic phase is realized in the ruthenate compound $\mathrm{Sr}_{3} \mathrm{Ru}_{2} \mathrm{O}_{7}$ [11]. Signatures of a nematic instablility have also been observed in cuprates [12] and other strongly correlated electron materials.

The nematic transition was found to be generically of first order at zero and sufficiently low temperatures for a number of models solved within the mean-field approximation 13 15], while the transition to the normal metallic state becomes continuous only for higher temperatures, above a tricritical temperature. In a phase diagram spanned by the chemical potential and temperature, the nematic phase resides within a dome-shaped region around van Hove filling. Due to the vicinity of the van Hove singularity in the density of states, at zero temperature the Landau expansion of the mean-field potential in powers of the order parameter $\phi$ exhibits negative interactions at any finite order in $\phi$. Hence, fluctuation effects cannot be computed perturbatively. This problem was recently overcome by using non-perturbative flow equations derived within the functional renormalization group (RG) framework, and it was shown that order-parameter fluctuations may actually suppress the first order character of the transition even at $T=0$, such that a continuous quantum phase transition accompanied by quantum critical fluctuations emerges [16].

In this letter we show that the impact of quantum fluctuations can be even more drastic. We show that the nematic order present within MFT can be completely swept out by the order-parameter fluctuations. We also observe that one can tune the system to a scenario where a singular quantum critical point is present, while no ordered phase occurs whatsoever.

We analyse a system of itinerant electrons on a square lattice with a Hamiltonian of the form [17]

$$
H=\sum_{\mathbf{k}} \epsilon_{\mathbf{k}} n_{\mathbf{k}}+\frac{1}{2 L} \sum_{\mathbf{k}, \mathbf{k}^{\prime}, \mathbf{q}} f_{\mathbf{k k}^{\prime}}(\mathbf{q}) n_{\mathbf{k}}(\mathbf{q}) n_{\mathbf{k}^{\prime}}(-\mathbf{q})
$$


featuring the $d$-wave forward scattering interaction

$$
f_{\mathbf{k k}^{\prime}}(\mathbf{q})=-g(\mathbf{q}) d_{\mathbf{k}} d_{\mathbf{k}^{\prime}}
$$

where $d_{\mathbf{k}}=\cos k_{x}-\cos k_{y}$ is a form factor with $d_{x^{2}-y^{2}}$ symmetry. The coupling function $g(\mathbf{q}) \geq 0$ has a maximum at $\mathbf{q}=\underline{0}$ and is restricted to small momentum transfers by a momentum cutoff. We introduced $n_{\mathbf{k}}(\mathbf{q})=\sum_{\sigma} c_{\mathbf{k}-\mathbf{q} / 2, \sigma}^{\dagger} c_{\mathbf{k}+\mathbf{q} / 2, \sigma}$, where $c_{\mathbf{k} \sigma}^{\dagger}\left(c_{\mathbf{k} \sigma}\right)$ creates (annihilates) an electron with momentum $\mathbf{k}$ and spin orientation $\sigma$, and $L$ denotes the number of lattice sites. Note that $n_{\mathbf{k}}=n_{\mathbf{k}}(\underline{0})$. We consider a dispersion given by

$$
\epsilon_{\mathbf{k}}=-2 t\left(\cos k_{x}+\cos k_{y}\right)-4 t^{\prime} \cos k_{x} \cos k_{y}-2 t^{\prime \prime}\left(\cos 2 k_{x}+\cos 2 k_{y}\right)
$$

with nearest, next-nearest, and third-nearest hopping amplitudes $t, t^{\prime}$, and $t^{\prime \prime}$, respectively.

For sufficiently large $g=g(\underline{0})$ the interaction drives a $d$-wave Pomeranchuk instability leading to a nematic state with broken orientation symmetry, which can be described by the order parameter

$$
\phi=\frac{g}{L} \sum_{\mathbf{k}} d_{\mathbf{k}}\left\langle n_{\mathbf{k}}\right\rangle
$$

Introducing a fluctuating order parameter field via a Hubbard-Stratonovich transformation, the fermionic interaction term in Eq. (11) is decoupled, and the fermionic degrees of freedom can be integrated out. Retaining only the leading momentum and frequency dependencies of the two-point function and neglecting such dependencies in the higher-order vertex functions [18], one obtains the following action:

$$
\mathcal{S}[\phi]=\frac{T}{2} \sum_{\omega_{n}} \int \frac{d^{2} q}{(2 \pi)^{2}} \phi_{\mathbf{q}, \omega_{n}}\left(A_{0} \frac{\left|\omega_{n}\right|}{|\mathbf{q}|}+Z_{0} \mathbf{q}^{2}\right) \phi_{-\mathbf{q},-\omega_{n}}+\mathcal{U}[\phi],
$$

where $\phi_{\mathbf{q}, \omega_{n}}$ is the momentum representation of the order parameter field $\phi$, and $\omega_{n}=2 \pi n T$ with integer $n$ denotes the (bosonic) Matsubara frequencies. The momenta and frequencies contributing to the action $\mathcal{S}[\phi]$ are restricted by an ultraviolet cutoff $\Lambda_{0}$ to the region $A_{0} \frac{\left|\omega_{n}\right|}{|\mathbf{q}|}+$ $Z_{0} \mathbf{q}^{2} \leq Z_{0} \Lambda_{0}^{2}$. In the fermionic representation, $\Lambda_{0}$ is related to the maximal momentum transfer allowed by the interaction in Eq. (1). The values of the prefactors $A_{0}$ and $Z_{0}$ are related to the $d$-wave particle-hole bubble in presence of a (mean-field) order parameter $\phi_{0}$, and $Z_{0}$ depends also on the specific form of the function $g(\mathbf{q})$, as discussed in Refs. [16, 19]. We do not treat a possible dependence of $A_{0}$ and $Z_{0}$ on the direction of $\mathbf{q}$, such that the values we insert should be viewed as an angular average. The effective potential $\mathcal{U}[\phi]$ is 
given by

$$
\mathcal{U}[\phi]=\int_{0}^{\frac{1}{T}} d \tau \int d^{2} r U(\phi(\mathbf{r}, \tau))
$$

where

$$
U(\phi)=\frac{\phi^{2}}{2 g}-2 T \int \frac{d^{2} k}{(2 \pi)^{2}} \ln \left(1+e^{-\left(\epsilon_{\mathbf{k}}-\phi d_{\mathbf{k}}-\mu\right) / T}\right) .
$$

An action of the form (5) was already considered in Ref. [16]. A polynomial expansion of $U(\phi)$ yields negative coefficients at any order in $\phi$ and is therefore inadequate here. Our calculations are carried out within the one-particle irreducible version of the functional RG [20]. The analysis amounts to solving the flow equations for $U(\phi)$ and $Z$ derived in Ref. [16]. Here we only remark that we employ a truncation of the functional RG based on the approximation that the effective action retains the local structure given by Eq. (6). We do not impose any ansatz for $U(\phi)$ and therefore keep track of the renormalization of infinitely many couplings. The flow of the coefficient $Z$ captures the anomalous dimension of the order parameter field at criticality. The truncation level is analogous to the next-toleading order in the derivative expansion, which was developed in the context of classical criticality [21, 22].

We now present our results, which are obtained from the solution of the RG flow equations for the following set of parameters: $t=1, t^{\prime}=-1 / 6, t^{\prime \prime}=1 / 5, g=1 / 2, A_{0}=1, Z_{0}=10$, and for different values of $\Lambda_{0}$. For this choice of hoppings the density of states is strongly asymmetric and the chemical potential corresponding to the maximal $T_{c}$ is separated from the van Hove energy $\left(\mu_{\mathrm{vH}}=-1.444\right)$. The relatively large value of $Z_{0}$ corresponds to a pronounced peak of $g(\mathbf{q})$ at $\mathbf{q}=0$, which favors the nematic instability compared to instabilities at a finite $\mathbf{q}$. Fluctuations reduce the magnitude of the order parameter compared to the mean-field value, as expected, and the nematic region in the phase diagram shrinks (see Fig. 1). The suppression is naturally stronger for larger cutoffs $\Lambda_{0}$. For sufficiently large $\Lambda_{0}$ the nematic phase is located to the right of the van Hove filling. When $\Lambda_{0}$ exceeds a critical value $\Lambda_{0}^{*}$, no ordering occurs whatsoever. By continuity, for $\Lambda_{0}=\Lambda_{0}^{*}$ the phase diagram exhibits an isolated quantum critical point at $\mu=\mu_{\mathrm{qc}}^{*}$ and $T=0$, while the system is disordered in the entire $(\mu, T)$ plane. In the vicinity of the point $\left(\mu=\mu_{\mathrm{qc}}^{*}, T=0\right)$ the system displays soft fluctuations characterized by a large correlation length. For the present choice of parameters we find $\mu_{\mathrm{qc}}^{*} \approx-1.404$ and $\Lambda_{0}^{*} \approx e^{-0.2574}$. Note that for smaller values of $g$ the order would be destroyed already at smaller $\Lambda_{0}$. 

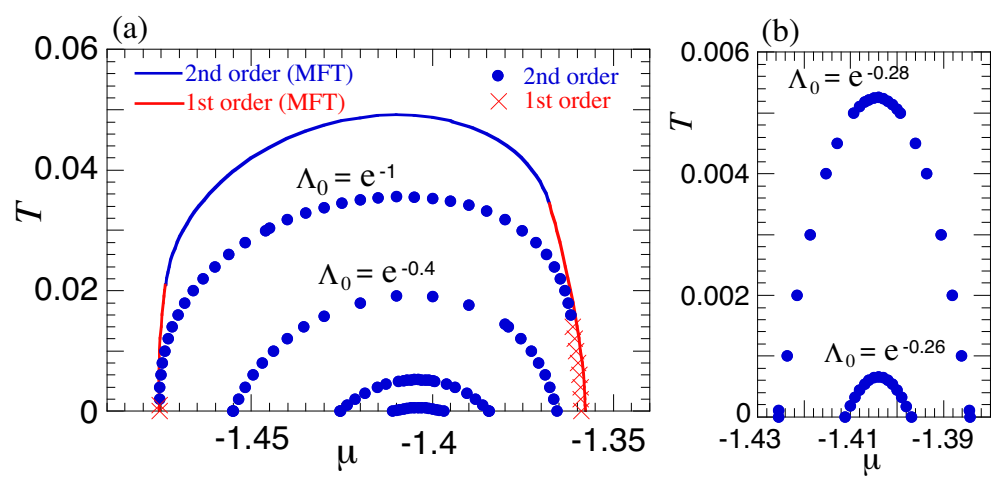

FIG. 1: (color online). Critical temperature for the nematic transition as a function of the chemical potential $\mu$. Left panel (a) Critical temperature in mean-field theory (MFT) and in the presence of fluctuations with four different choices of the ultraviolet (momentum transfer) cutoff, $\Lambda_{0}=$ $e^{-1}, e^{-0.4}, e^{-0.28}, e^{-0.26}$. Right panel (b) Enlargement of the results for $\Lambda_{0}=e^{-0.28}, e^{-0.26}$.

We have also computed the behavior of the correlation length $\xi$ upon approaching the quantum critical points at the edges of the nematic dome for $\Lambda_{0}<\Lambda_{0}^{*}$, and for the isolated quantum critical point in the special case $\Lambda_{0}=\Lambda_{0}^{*}$. The correlation length in the symmetric phase is determined by the curvature of the potential $U^{\prime \prime}(0)$ and the renormalization constant $Z$ at the end of the flow via $\xi^{2}=Z / U^{\prime \prime}(0)$. For $\Lambda_{0}<\Lambda_{0}^{*}$ the results are consistent with the predictions of Hertz-Millis theory [23] for a two-dimensional system with a dynamical exponent $z=3$, namely $\xi^{2} \sim|T \log T|^{-1}$ at fixed $\mu=\mu_{\mathrm{qc}}$ and $\xi^{2} \sim\left|\mu-\mu_{\mathrm{qc}}\right|^{-1}$ at $T=0$.

The scenario occurring for $\Lambda_{0}=\Lambda_{0}^{*}$ requires fine-tuning. However, features resembling quantum-critical behavior persist also for $\Lambda_{0}>\Lambda_{0}^{*}$. In Fig. 2 we plot the correlation length $\xi$ as a function of $\mu$ at $T=0$ for a sequence of values of $\Lambda_{0}$ approaching $\Lambda_{0}^{*}$ from above. The peak formed around $\mu=\mu_{\mathrm{qc}}^{*}$ becomes rapidly pronounced as $\Lambda_{0} \rightarrow \Lambda_{0}^{*}$. Remarkably, although the quantum critical point occurring for $\Lambda_{0}=\Lambda_{0}^{*}$ is Gaussian, the exponent $\nu$ describing the divergence of $\xi$ at $\mu \rightarrow \mu_{\mathrm{qc}}^{*}$ takes the value 1 instead of the usual value $\frac{1}{2}$. This is easily understood by noticing that for $\Lambda_{0}=\Lambda_{0}^{*}$ the quadratic mass term in the Landau expansion must not take negative values in the absence of an ordered phase and therefore cannot be proportional to $\mu-\mu_{\mathrm{qc}}^{*}$ as in the usual cases. The natural $\mu$-dependence of the quadratic term is therefore quadratic in $\mu-\mu_{\mathrm{qc}}^{*}$ near $\mu_{\mathrm{qc}}^{*}$, which leads immediately to $\nu=1$. This peculiarity does not influence the behavior when the singularity is cut off by temperature. In fact for fixed $\mu=\mu_{\mathrm{qc}}^{*}$ we recover the usual [23] behavior $\xi \sim|T \log T|^{-1 / 2}$, 

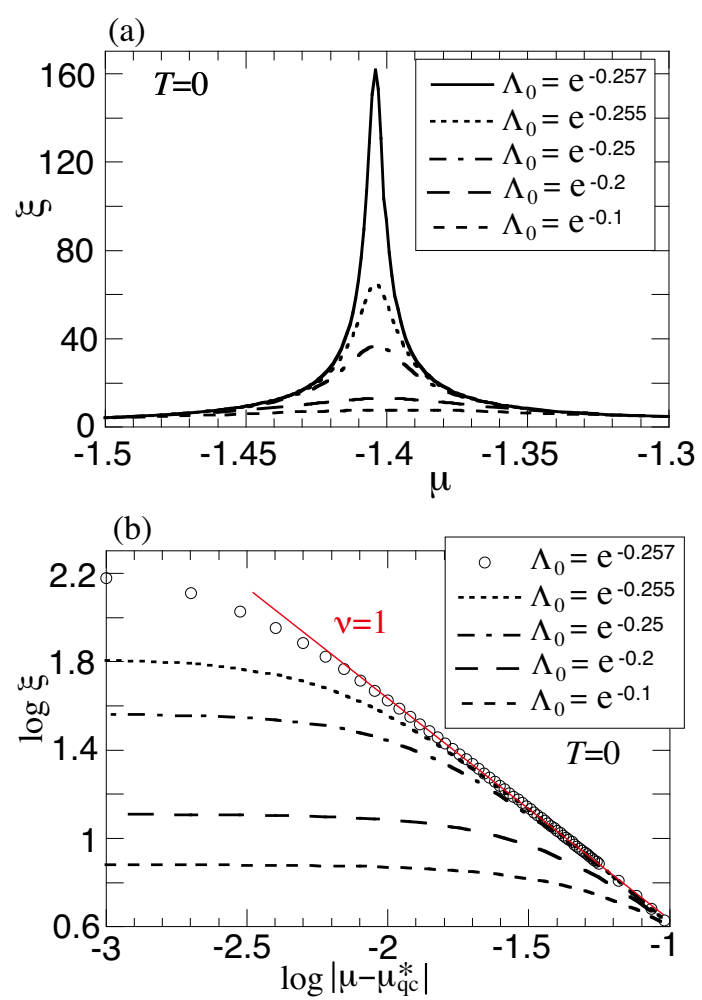

FIG. 2: (color online) Top (a) Correlation length $\xi$ as a function of $\mu$ at $T=0$ for various values of $\Lambda_{0}>\Lambda_{0}^{*}$. Bottom (b) Double logarithmic plot of $\xi$ versus $\mu-\mu_{\mathrm{qc}}^{*}$ and fit to the exponent $\nu=1$.

as shown in Fig. 3. For $\Lambda_{0}>\Lambda_{0}^{*}$ no singular point in the phase diagram occurs, but the

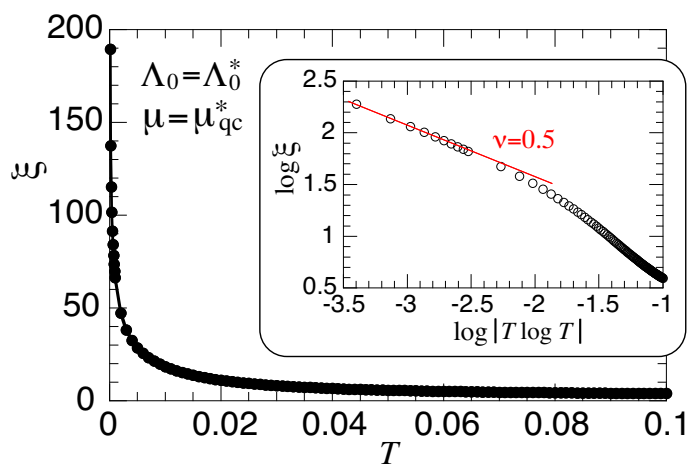

FIG. 3: (color online) Temperature dependence of the correlation length $\xi$ for the special case $\mu=$ $\mu_{\mathrm{qc}}^{*}$ and $\Lambda_{0}=\Lambda_{0}^{*}$. Inset: Double logarithmic plot exhibiting the expected behavior $\xi \sim|T \log T|^{-1 / 2}$ for small $T$.

correlation length takes sizable values in the vicinity of the point $\left(\mu=\mu_{\mathrm{qc}}^{*}, T=0\right)$ as long as $\Lambda_{0}$ is not significantly larger than $\Lambda_{0}^{*}$. 
One may be surprised to find the nematic order fully eliminated by fluctuations even at van Hove filling. In MFT, the diverging density of states at van Hove filling leads to an instability of the symmetric phase at any (arbitrarily small) coupling $g$ [15]. One would expect this to remain true also beyond MFT, provided the system remains a Fermi liquid. Inversely, the absence of nematic order at van Hove filling despite the presence of a $d$-wave attraction $g$ would imply the absence of fermionic quasi-particles, that is, non-Fermi liquid behavior. Unlike the situation at the quantum critical point, that non-Fermi liquid behavior is not related to critical fluctuations. Working with an effective order parameter action we do not have direct access to fermionic properties. However, the breakdown of Fermi liquid theory at van Hove points was already diagnosed many years ago by Dzyaloshinskii [24]. In the light of that work the complete suppression of order by fluctuations even at van Hove filling is a consistent possibility.

In summary, we have analyzed a two-dimensional interacting electron system which exhibits a nematic phase in mean-field theory. We have shown that fluctuations can extinguish the nematic order completely from the phase diagram spanned by the chemical potential and temperature. For a special choice of parameters a quantum critical point can be realized in the absence of order. Despite being realized as a consequence of strong quantum fluctuations, the quantum critical point itself is Gaussian. The temperature dependence of the correlation length in the quantum critical regime above this isolated quantum critical point follows the conventional behavior known for Gaussian quantum criticality. However, at zero temperature the correlation length diverges with an exponent $\nu=1$ instead of $\nu=\frac{1}{2}$ upon approaching the critical chemical potential. While the present calculation was carried out for a specific interaction triggering a nematic phase, an analysis of fluctuation effects in other two-dimensional systems described by an action of the form (15) can be performed along the same lines.

We are grateful to J. Bauer, K. Byczuk, T. Holder, and C. Husemann for valuable discussions. This work was supported by the German Research Foundation through the research group FOR 723. H.Y. was also supported by a Grant-in-Aid for Scientific Research from Monkasho. 
[1] S. Sachdev, Quantum Phase Transitions (Cambridge University Press, Cambridge, UK, 1999).

[2] M. Vojta, Rep. Prog. Phys. 66, 2069 (2003).

[3] H. v. Löhneysen et al., Rev. Mod. Phys. 79, 1015 (2007).

[4] N.D. Mermin, H. Wagner, Phys. Rev. Lett. 17, 1133 (1966).

[5] D. Belitz, T.R. Kirkpatrick, and T. Vojta, Rev. Mod. Phys. 77, 579 (2005).

[6] F. Fucito and G. Parisi, J. Phys. A 14, L499 (1981).

[7] R.L. Greenblatt, M. Aizenman, and J.L. Lebowitz, Phys. Rev. Lett. 103, 197201 (2009).

[8] H. Yamase and H. Kohno, J. Phys. Soc. Jpn. 69, 332 (2000); ibid 69, 2151 (2000).

[9] C.J. Halboth and W. Metzner, Phys. Rev. Lett. 85, 5162 (2000).

[10] E. Fradkin et al., Annu. Rev. Condens. Matter Phys. 1, 153 (2010).

[11] S.A. Grigera et al., Science 306, 1154 (2004); R.A. Borzi et al., Science 315, 214 (2007); A.W. Rost et al., Science 325, 1360 (2009).

[12] Y. Ando et al., Phys. Rev. Lett. 88, 137005 (2002); V. Hinkov et al., Nature (London) 430, 650 (2004); Y. Kohsaka et al., Science 315, 1380 (2007); V. Hinkov et al., Science 319, 597 (2008); R. Daou et al., Nature (London) 463, 519 (2010).

[13] H.-Y. Kee, E.H. Kim, and C.-H. Chung, Phys. Rev. B 68, 245109 (2003).

[14] I. Khavkine et al., Phys. Rev. B 70, 155110 (2004).

[15] H. Yamase, V. Oganesyan, and W. Metzner, Phys. Rev. B 72, 035114 (2005).

[16] P. Jakubczyk, W. Metzner, and H. Yamase, Phys. Rev. Lett. 103, 220602 (2009).

[17] W. Metzner, D. Rohe, and S. Andergassen, Phys. Rev. Lett. 91, 066402 (2003).

[18] J. Hertz, Phys. Rev. B 14, 1165 (1976).

[19] L. Dell'Anna and W. Metzner, Phys. Rev. B 73, 045127 (2006).

[20] C. Wetterich, Phys. Lett. B 301, 90 (1993).

[21] J. Berges, N. Tetradis, and C. Wetterich, Phys. Rep. 363, 223 (2002).

[22] B. Delamotte, D. Mouhanna, and M. Tissier, Phys. Rev. B 69, 134413 (2004); B. Delamotte, arXiv:cond-mat/0702365.

[23] A. J. Millis, Phys. Rev. B 48, 7183 (1993).

[24] I. Dzyaloshinskii, J. Phys. I France 6, 119 (1996). 